\title{
A broadband polarization splitter directional coupler based on tilted subwavelengh grating metamaterials
}

\author{
José Manuel Luque-González ${ }^{1, *}$, Alaine Herrero-Bermello², Alejandro Ortega-Moñux ${ }^{1}$, Marina Sánchez-Rodríguez ${ }^{1}$, Aitor V. \\ Velasco $^{2}$, Jens H. Schmid ${ }^{3}$, Pavel Cheben ${ }^{3,4}$, Íñigo Molina-Fernández1,5, Robert Halir ${ }^{1,5}$ \\ ${ }^{1}$ Universidad de Málaga, Departamento de Ingeniería de Comunicaciones, ETSI Telecomunicación, Málaga 29071, Spain \\ ${ }^{2}$ Instituto de Óptica Daza de Valdés, Consejo Superior de Investigaciones Científicas (CSIC), Madrid 28006, Spain \\ ${ }^{3}$ National Research Council Canada, 1200 Montreal Road, Bldg. M50, Ottawa K1A OR5, Canada \\ ${ }^{4}$ Center for Research in Photonics, University of Ottawa, Ottawa K1N6N5, Canada \\ ${ }^{5}$ Bionand Center for Nanomedicine and Biotechnology, Parque Tecnológico de Andalucía, Málaga 29590, Spain \\ *jmlg@ic.uma.es
}

\begin{abstract}
Tilted subwavelength gratings (SWG) allows anisotropy tailorable metamaterials with applications in polarization management. Based on this concept, here we experimentally demonstrate a broadband directional-couplerbased polarization beam splitter with sub-decibel insertion loss in a $100 \mathrm{~nm}$ bandwidth.
\end{abstract}

Keywords- silicon photonics, silicon-on-insulator subwavelength gratings, metamaterials, polarization management

\section{INTRODUCTION}

Directional couplers are often used for polarization beam splitting in highly birefringent photonic integration platforms such as silicon-on-insulator (SOI) [1-5]. These devices can be classified in two types: symmetric and asymmetric directional coupler polarization beam splitters (DC-PBS). In symmetric DC-PBSs, both waveguides are equal, therefore, the phase matching condition is satisfied for both polarizations at the same time. In this situation a proper design of the TE and TM coupling lengths, $L_{\pi}^{\mathrm{TE}}$ and $L_{\pi}^{\mathrm{TM}}$, is required to achieve the polarization splitting condition: $m L_{\pi}^{\mathrm{TE}}=n L_{\pi}^{\mathrm{TM}}$, where $m$ and $n$ are integers and $|m-n|$ is an odd number. This results in comparatively long devices sensitive to fabrication imperfections since the two polarizations are affected differently. On the contrary, in asymmetric DC-PBS only one of the polarizations satisfy the phase matching condition, yielding shorter and robust, making it challenging to achieve the required asymmetry.

In this work we propose a novel method to break the symmetry in a DC-PBS by using lithography tailorable metamaterials implemented with subwavelength grating waveguides [6-7]. Our device [Fig. 1(a)] comprises two fully etched SWG waveguides which only differs in the tilt angle of the silicon segments, and a central silicon strip which optimizes the device bandwidth. The tilt angle only minimally affects the TM polarization [8], so that the TM mode still couples to the cross port [Fig. 1(b)]. On the contrary, the tilt angle strongly changes the equivalent index for TE polarization, thus suppressing power transfer to the cross waveguide [Fig. 1(c)].

\section{DEVICE DESIGN}

According to the coupled mode theory [9], the power transferred between two parallel waveguides is inversely proportional to the difference between the effective indices of the mode of each waveguide, $\Delta \mathrm{n}_{\text {eff }}$. For our design we consider a DC-PBS with two SWG waveguides separated by a distance $s=0.5 \mu \mathrm{m}$. Both waveguides have initially the same width $w=0.6 \mu \mathrm{m}$ and the same period $\Lambda_{0}=0.275$
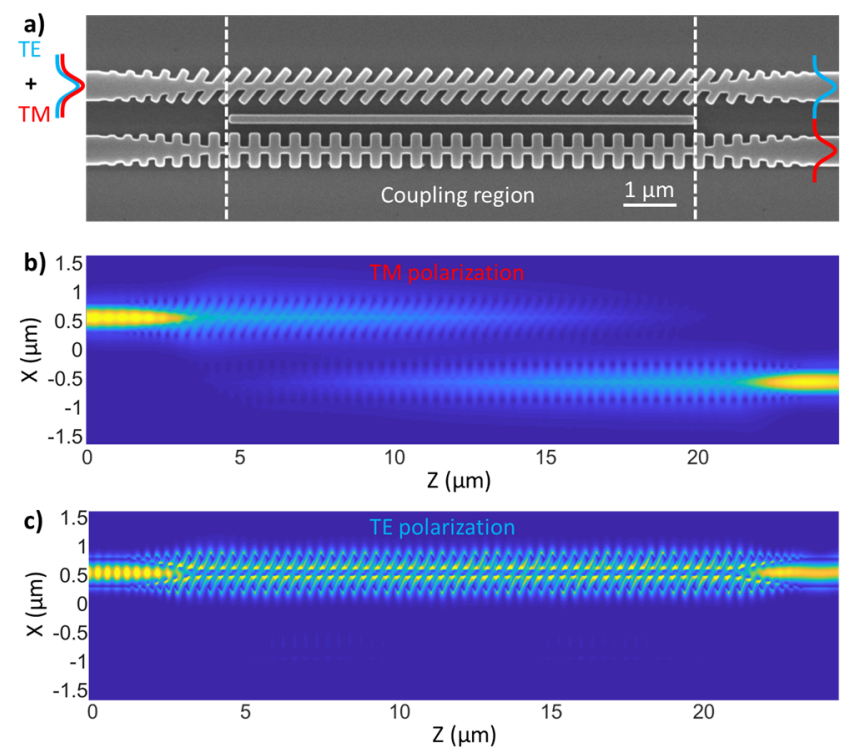

Figure 1. Scanning Electron Microscope image of the polarization beam splitter directional coupler (before $\mathrm{SiO}_{2}$ cladding deposition). (b), (c) Simulation of the electromagnetic field propagation along the proposed structure for (b) TM and (c) TE polarizations.

$\mu \mathrm{m}$ when the tilt angle is $\theta=0^{\circ}$, with a duty cycle $\mathrm{DC}=0.5$. Fig. 2 shows the effective index difference as a function of the tilt angle for the proposed DC-PBS. From this figure it is clear that the TE polarization effective index difference increases with the tilt angle while it remains almost unaltered for the TM polarization. Thereby, the power transfer of the TE polarization is reduced while it is almost unchanged for TM polarization. In our design, a tilt angle of $\theta=40^{\circ}$ has been chosen. The bandwidth of the device is mainly limited by the TM polarization, since for this polarization the

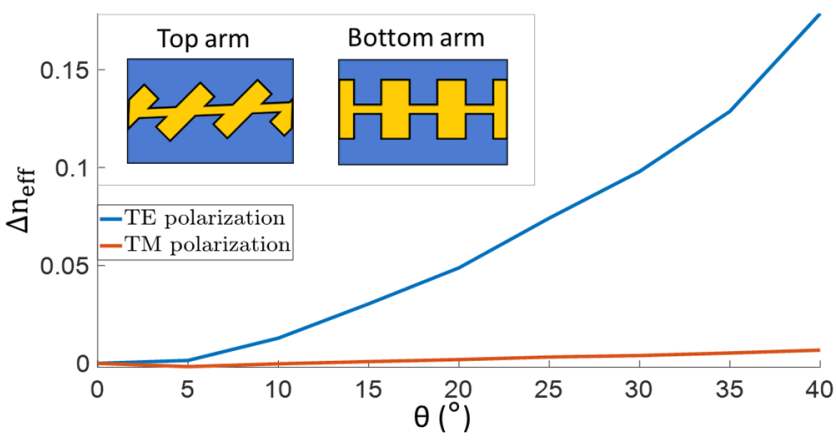

Figure 2 (a) Effective index difference between the top and the bottom waveguide of the directional coupler as a function of the tilt angle for both polarizations. 
device acts as a directional coupler. The bandwidth of a directional coupler is mainly governed by the wavelength variation of the coupling length: $L_{\pi}^{T M}(\lambda)=\lambda /\left[2\left(n_{1}^{T M}(\lambda)-\right.\right.$ $\left.\left.n_{2}^{T M}(\lambda)\right)\right]$ where $n_{1,2}^{T M}$ are the effective indices of the even and the odd TM polarized supermodes. As this wavelength variation is, to first order, proportional to the square of the coupling length, a simple way to enhance the bandwidth of the device is to reduce its coupling length. To do so, here we insert a central silicon strip between the two arms of the directional coupler [10-11]. This central silicon strip increases the effective index of the even supermode, $n_{1}^{T M}(\lambda)$, without affecting the odd mode, $n_{2}^{T M}(\lambda)$. As the coupling length is inversely proportional to the difference between the effective indices, the central silicon strip helps to shorten the coupling length, thereby, reducing the wavelength dependence. In Figure 3 we show the device performance for an optimized width of $150 \mathrm{~nm}$ for the silicon strip. The main figures of merit are the insertion loss (IL) and the extinction ratio (ER): $\mathrm{IL}^{\mathrm{TE}}=$ $\mathrm{P}_{\mathrm{bar}}^{\mathrm{TE}} / \mathrm{P}_{\text {in }}^{\mathrm{TE}} ; \quad \mathrm{IL}^{\mathrm{TM}}=\mathrm{P}_{\text {cross }}^{\mathrm{TM}} / \mathrm{P}_{\text {in }}^{\mathrm{TM}} \quad, \quad \mathrm{ER}^{\mathrm{TE}}=\mathrm{P}_{\text {cross }}^{\mathrm{TE}} /$ $\mathrm{P}_{\mathrm{bar}}^{\mathrm{TE}} ; \quad \mathrm{ER}^{\mathrm{TM}}=\mathrm{P}_{\mathrm{bar}}^{\mathrm{TM}} / \mathrm{P}_{\text {cross }}^{\mathrm{TM}}$ where $\mathrm{P}_{\text {bar }}^{\mathrm{TE} / \mathrm{TM}}$ and $\mathrm{P}_{\text {cross }}^{\mathrm{TE} / \mathrm{TM}}$ is the power in the fundamental TE/TM mode in the bar and cross ports, and $\mathrm{P}_{\text {in }}^{\mathrm{TE} / \mathrm{TM}}$ is the power of the fundamental TE/TM mode the input port. The optimized device has an extinction ratio $>20 \mathrm{~dB}$ and insertion loss $<1 \mathrm{~dB}$ over a bandwidth of 86 $\mathrm{nm}$.

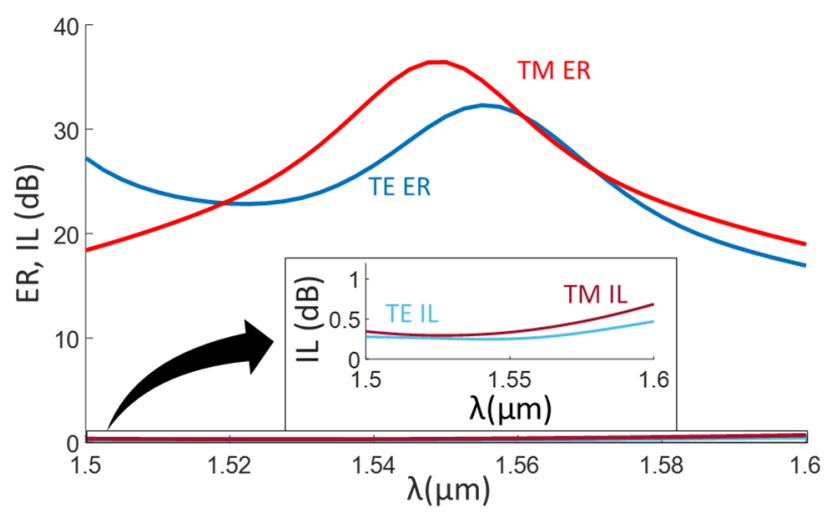

Figure 3. Simulated extinction ratio and insertion loss for the TE and TM polarizations of the tilted SWG directional coupler.

\section{EXPERIMENTAL RESULTS}

The device has been fabricated in a $220 \mathrm{~nm}$ SOI platform with a $2-\mu \mathrm{m}$-thick buried oxide (BOX) and a $2.2-\mu \mathrm{m}$-thick $\mathrm{SiO}_{2}$ upper cladding [12]. Figure 4 shows the measured insertion loss and extinction ratio of the nominal device. The fabricated nominal device achieves an extinction ratio in excess of $15 \mathrm{~dB}$ and an insertion loss below $1 \mathrm{~dB}$, for both polarizations over a $72 \mathrm{~nm}$ bandwidth $(1505-1577 \mathrm{~nm})$.

\section{CONCLUSIONS}

In this work we have proposed and experimentally demonstrated a new type of directional coupler based polarization splitter, by independently controlling the TE and TM phase matching conditions using SWG metamaterial anisotropy engineering. Our device incorporates tilted subwavelength grating structures to break the symmetry of the directional coupler. We believe that this new concept of symmetry engineering opens promising venues for polarization control in integrated optical devices.

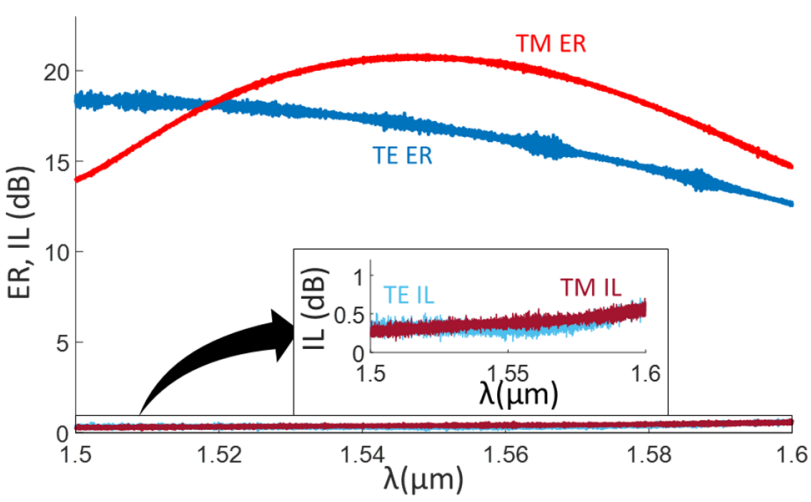

Figure 4. Measured extinction ratio and insertion loss of the nominal device. The IL is below $1 \mathrm{~dB}$ and the ER is better than $15 \mathrm{~dB}$ in a bandwidth of 72 nm.

\section{ACKNOWLEDGMENT}

Universidad de Málaga; Ministerio de Economía y Competitividad (MINECO) (TEC2016-80718-R); Ministerio de Educación, Cultura y Deporte (MECD) (FPU16/06762), Fondo Europeo de Desarrollo Regional-FEDER, Proyecto I+D+i en el marco del Programa Operativo FEDER Andalucía 2014-2020 (UMA18-FEDERJA-219), Spanish Ministry of Science, Innovation and Universities (MICINN) under grants CDTI SNEO-20181232 (Alcyon Photonics S.L.) and RTI2018-097957-B-C33; Community of Madrid - FEDER funds (S2018/NMT-4326); Horizon 2020 research and innovation program under the Marie Sklodowska-Curie grant No. 734331.

\section{REFERENCES}

[1] D. Dai and J. E. Bowers, "Novel concept for ultracompact polarization splitter-rotator based on silicon nanowires," Opt. Express 19, 10940 (2011).

[2] J. Wang, D. Liang, Y. Tang, D. Dai, and J. E. Bowers, "Realization of an ultra-short silicon polarization beam splitter with an asymmetrical bent directional coupler," Opt. Lett. 38, 4 (2013).

[3] F. Zhang, H. Yun, Y. Wang, Z. Lu, L. Chrostowski, and N. A. F. Jaeger, "Compact broadband polarization beam splitter using a symmetric directional coupler with sinusoidal bends," Opt. Lett. 42, 235 (2017).

[4] B. Ni and J. Xiao, "Ultracompact and Broadband Silicon-Based Polarization Beam Splitter Using an Asymmetrical Directional Coupler," IEEE J. Quantum Electron. 53, 1 (2017).

[5] Y. Tian, J. Qiu, C. Liu, S. Tian, Z. Huang, and J. Wu, "Compact polarization beam splitter with a high extinction ratio over $\mathrm{S}+\mathrm{C}+\mathrm{L}$ band," Opt. Express 27, 999 (2019).

[6] R. Halir, A. Ortega-Moñux, D. Benedikovic, G. Z. Mashanovich, J. G. Wangüemert-Pérez, J. H. Schmid, Í. Molina-Fernández, and P. Cheben, "Subwavelength-grating metamaterial structures for silicon photonic devices," Proc. IEEE, 106, 2144 (2018).

[7] P. Cheben, R. Halir, J. H. Schmid, H. A. Atwater, and D. Smith, "Subwavelength integrated photonics," Nature, 560, 565 (2018).

[8] J. M. Luque-González, A. Herrero-Bermello, A. Ortega-Moñux, Í. Molina-Fernández, A. V. Velasco, P. Cheben, J. H. Schmid, S. Wang, and R. Halir, "Tilted subwavelength gratings: controlling anisotropy in metamaterial nanophotonic waveguides," Opt. Lett., 43, 4691 (2018).

[9] C.-L. Chen, Foundations for Guided-Wave Optics (John Wiley \& Sons, Inc., 2006).

[10] D. W. Kim, M. H. Lee, Y. Kim, and K. H. Kim, "Planar-type polarization beam splitter based on a bridged silicon waveguide coupler," Opt. Express 23, 998 (2015).

[11] Y. Kim, M. H. Lee, Y. Kim, and K. H. Kim, "High-extinction-ratio directional-coupler-type polarization beam splitter with a bridged silicon wire waveguide," Opt. Lett. 43, 3241 (2018).

[12] "Applied Nanotools Inc. Canada," https://www.appliednt.com/nanosoi/. 